Real Analysis Exchange

Vol. 23(2), 1997-1998, pp. 799-803

Chew Tuan-Seng, Wong Woon-Kwong and Tan Geok-Choo

Department of Mathematics, National University of Singapore, Singapore

119260, e-mail: matcts@nus.edu.sg

\title{
ON ABSOLUTELY HENSTOCK INTEGRABLE FUNCTIONS
}

\begin{abstract}
In this note, we shall prove that every absolutely Henstock integrable function is McShane integrable, without using the measurability of Henstock integrable functions and gauge functions.
\end{abstract}

The result that every absolutely Henstock integrable function is McShane integrable is well-known. In $[1,2]$, the proof has been simplified without using Egoroff's theorem and truncated functions. In this note, we shall further simplify the proof in [1] without using the measurability of Henstock integrable functions and gauge functions. It has the advantage that, in a more general setting, the measurability of integrable functions and gauge functions may not make sense or its verification may be very involved. Lemma 2 in this note is crucial, though the proof is elementary.

By an interval $E$ in $\mathbf{R}^{m}$, we mean the 'box' $\left[a_{1}, b_{1}\right] \times\left[a_{2}, b_{2}\right] \times \cdots\left[a_{m}, b_{m}\right]$, where $a_{i}, b_{i} \in \mathbf{R}$ for $i=1,2, \cdots, m$. We denote the volume of $E$ by $|E|$. Given a fixed interval $E$ an elementary set is a subinterval of $E$ or a union of finite number of non-overlapping subintervals of $E$. We denote by $\mathcal{B}$ the collection of open sets whose complement with respect to $E$ is an elementary set or an empty set. Given a $\xi \in E$ and a positive $\delta$, we let $B(\xi, \delta)=\{x \in E:|x-\xi|<$ $\delta\}$, that is the ball centered at $\xi$ and radius $\delta$.

A real-valued function $f$ defined on $E$ is said to be Henstock integrable to $F(E)$ if for every $\epsilon>0$, there is a $\delta(\xi)>0$ such that for any $\delta$-fine full division $D=\{(I, \xi)\}$ of $E$, i.e. $\xi \in I \subseteq B(\xi, \delta(\xi))$, we have

$$
\left|(D) \sum f(\xi)\right| I|-F(E)|<\epsilon .
$$

It can be showed that whenever $f$ is Henstock integrable on $E$, it is Henstock integrable on any subinterval $I$ of $E$. A function $f$ is said to be absolutely Henstock integrable on $E$ if both $f$ and $|f|$ are Henstock integrable on $E$.

Key Words: Henstock, McShane, Absolutely Henstock

Mathematical Reviews subject classification: 26A39

Received by the editors December 15, 1997 
A real-valued function $f$ defined on $E$ is said to be $M c$ Shane integrable to $M$ if for every $\epsilon>0$, there is a $\delta(\xi)>0$ such that for any $\delta$-fine full division $D=\{(I, \xi)\}$ of $E$, i.e. $I \subseteq B(\xi, \delta(\xi))$, but $\xi$ does not necessarily belong to $I$, we have

$$
\left|(D) \sum f(\xi)\right| I|-M|<\epsilon \text {. }
$$

The following lemma can be proved readily.

Lemma 1. Let $f$ be an Henstock integrable function on $E$. Then $G \in \mathcal{B}$ implies $f$ is Henstock integrable on $G$.

Lemma 2. Let $f$ be non-negative, Henstock integrable on $E$ with $F(I)=\int_{I} f$, $I$ is any subinterval of $E$. Let $X$ be any subset of $E$. Then for any $\epsilon>0$, there exists $G(\epsilon) \in \mathcal{B}$ with $X \subset G(\epsilon)$ such that whenever there is a finite collection $\left\{I_{1}, I_{2}, \cdots, I_{n}\right\}$ of non-overlapping subintervals of $E$ with $I_{i} \subseteq G(\epsilon)-X$ for each $i$, we have

$$
\sum_{i=1}^{n} F\left(I_{i}\right)<\epsilon
$$

Proof. Let $X \subset E$. Define the class $B^{*}=\{G \in \mathcal{B}: X \subset G\}$, which is non-empty as $E \in B^{*}$. Since $f$ is Henstock integrable on $E$, it is Henstock integrable on $G$, for each $G \in B^{*}$. Let $A=\inf \left\{\int_{G} f: G \in B^{*}\right\}$, which exists and $0 \leq A<\infty$. For a given $\epsilon>0$, there exists $G(\epsilon) \in B^{*}$ such that

$$
0 \leq \int_{G(\epsilon)} f-A<\epsilon / 2 .
$$

Suppose $\left\{I_{1}, I_{2}, \cdots, I_{n}\right\}$ is a collection of non-overlapping subintervals of $E$ with $I_{i} \subseteq G(\epsilon)-X$ for each $i$. Let $G^{\prime}=G(\epsilon)-\cup_{i=1}^{n} I_{i}$. Then $G^{\prime} \in B^{*}$. Moreover,

$$
0 \leq \int_{G^{\prime}} f-A \leq \int_{G(\epsilon)} f-A \leq \epsilon / 2 .
$$

Thus, $0 \leq \sum_{i=1}^{n} F\left(I_{i}\right)=\int_{G(\epsilon)} f-\int_{G^{\prime}} f<\epsilon$.

For an absolutely Henstock integrable function $f$, we have $\sum|F(I)| \leq$ $\sum G(I)$ where $G(I)=\int_{I}|f|$. Furthermore, the constant function $c(x) \equiv 1$ is Henstock integrable and $C(I)=\int_{I} c=|I|$. Thus, we have

Corollary 1. Suppose $f$ is an absolutely Henstock integrable function on $E$ with $F(I)=\int_{I} f$, where $I$ is any subinterval of $E$. Let $X$ be any subset of $E$. Then for any $\epsilon>0$, there exists $G(\epsilon) \in \mathcal{B}$ with $X \subset G(\epsilon)$ such that whenever 
there is a finite collection $\left\{I_{1}, I_{2}, \cdots, I_{n}\right\}$ of non-overlapping subintervals of $E$ with $I_{i} \subseteq G(\epsilon)-X$ for each $i$, we have

$$
\sum_{i=1}^{n}\left|F\left(I_{i}\right)\right|<\epsilon \text { and } \sum_{i=1}^{n}\left|I_{i}\right|<\epsilon .
$$

Theorem 1. If $f$ is absolutely Henstock integrable on $E$, then $f$ is McShane integrable on $E$.

Proof. The idea is similar to that of Theorem 1 in [1]. Let $\epsilon>0$ be given. Since $f$ is Henstock integrable, there is $\delta(\xi)$ such that $0<\delta(\xi) \leq 1$ and $(D) \sum|f(\xi)| I|-F(I)|<\epsilon$ whenever $D=\{(I, \xi)\}$ is a partial $\delta$-fine division of $E$, by Henstock's Lemma.

For each integer $k$, we define the set $X(\epsilon, k)=\{x \in E:(k-1) \epsilon \leq f(x)<$ $k \epsilon\}$ so that for any $x, x^{\prime}$ in $X(\epsilon, k)$, we have $\left|f(x)-f\left(x^{\prime}\right)\right|<\epsilon$.

Now, for each positive integer $n$ greater than or equal to 2 , we define the set $X(\epsilon, k, n)=\{x \in X(\epsilon, k): 1 / n<\delta(x) \leq 1 /(n-1)\}$.

Next, we divide $E$ into $p(n)$ subintervals $I(n, q)$, where $q=1,2, \cdots, p(n)$ such that $\operatorname{diag} I(n, q) \leq 1 / n$ where $\operatorname{diag} I$ is the diagonal of $I$. We denote by $X(\epsilon, k, n, q)$ the set $X(\epsilon, k, n) \cap I^{o}(n, q)$, where $I^{o}(n, q)$ is the interior of $I(n, q)$. We note that whenever $\xi \in X(\epsilon, k, n, q)$, we have $I(n, q) \subset B(\xi, \delta(\xi))$.

By Corollary 1, with $E$ replaced by $I(n, q)$, there exists $G(\epsilon, k, n, q)$ such that $X(\epsilon, k, n, q) \subset G(\epsilon, k, n, q) \subset I(n, q)$ and satisfying the property in Corollary 1 , with $\epsilon$ replaced by $\epsilon /(|k|+1) 2^{|k|+n+q}$.

We shall now define $\delta^{\prime}(\xi)$ for each $\xi \in E$. Note that $\xi \in X(\epsilon, k, n, q)$ or $\xi$ is on the boundary of $I(n, q)$ for some $(n, q)$. Let $S$ be the union of the boundary of all $I(n, q)$. We define $\delta^{\prime}$ on $S$ in such a way that for any $\delta^{\prime}$-fine partial division $D=\{(I, \xi)\}$, with $\xi \in S$, we have

$$
(D) \sum|f(\xi)| I||<\epsilon / 2 \text { and }(D) \sum|F(I)|<\epsilon / 2 .
$$

For $\xi \in X(\epsilon, k, n, q)$, we shall define $\delta^{\prime}(\xi)$ such that $0<\delta^{\prime}(\xi)<\delta(\xi)$ and $B\left(\xi, \delta^{\prime}(\xi)\right) \cap I(n, q) \subseteq G(\epsilon, k, n, q) \subset I(n, q)$.

Suppose we have a $\delta^{\prime}$-fine McShane division $D^{\prime}=\{(I, \xi)\}$ of $E$. If $\xi \notin I$ and $\xi \in X(\epsilon, k, n, q)$, then either $I \cap X(\epsilon, k, n, q)=\phi$ or $I \cap X(\epsilon, k, n, q) \neq \phi$. For the former, $I \subseteq G(\epsilon, k, n, q)-X(\epsilon, l, n, q)$ so that, summing over all such $I, \sum_{1}|F(I)|<\epsilon,\left|\sum_{1} f(\xi)\right| I||<\epsilon$, by Corollary 1 .

For $I$ with $I \cap X(\epsilon, k, n, q) \neq \phi$, there exists $\xi^{\prime} \in I \cap X(\epsilon, k, n, q)$. Hence we have

$$
\xi^{\prime} \in I \subset B\left(\xi, \delta^{\prime}(\xi)\right) \subset I(n, q) \subset B\left(\xi^{\prime}, \delta\left(\xi^{\prime}\right)\right)
$$


so that $\left(I, \xi^{\prime}\right)$ is $\delta\left(\xi^{\prime}\right)$-fine and $\xi^{\prime} \in I$. Summing over all $I$ with $I \cap X(\epsilon, k, n, q)$ $\neq \phi$, we get

$$
\begin{gathered}
\left|\sum_{2} f(\xi)\right| I|-F(I)| \leq\left|\sum_{2}\left(f(\xi)-f\left(\xi^{\prime}\right)\right)\right| I\left|+f\left(\xi^{\prime}\right)\right| I|-F(I)| \\
\leq \sum_{2}\left|\left(f(\xi)-f\left(\xi^{\prime}\right)\right)\right| I||+\left|\sum_{2} f\left(\xi^{\prime}\right)\right| I|-F(I)| .
\end{gathered}
$$

The full summation can now be done in the following way:

$$
\left|\left(D^{\prime}\right) \sum_{(I, \xi)} f(\xi)\right| I|-F(I)| \leq\left|\sum_{\xi \in S} f(\xi)\right| I|-F(I)|+\left|\sum_{\xi \notin S} f(\xi)\right| I|-F(I)| .
$$

By our choice of $\delta^{\prime}$, the first sum, $\left|\sum_{\xi \in S} f(\xi)\right| I|-F(I)| \leq \epsilon$, whereas for the second sum, we get

$$
\begin{aligned}
& \left|\sum_{\xi \notin S} f(\xi)\right| I|-F(I)| \leq\left|\sum_{\xi \in I} f(\xi)\right| I|-F(I)|+\left|\sum_{\xi \notin I} f(\xi)\right| I|-F(I)| \\
& \leq\left|\sum_{\xi \in I} f(\xi)\right| I|-F(I)|+\left|\sum_{1} f(\xi)\right| I|-F(I)|+\left|\sum_{2} f(\xi)\right| I|-F(I)| \\
& \leq\left|\sum_{\xi \in I} f(\xi)\right| I|-F(I)|+\left|\sum_{1} f(\xi)\right| I|-F(I)| \\
& +\left|\sum_{2}\left(f(\xi)-f\left(\xi^{\prime}\right)\right)\right| I\left|+f\left(\xi^{\prime}\right)\right| I|-F(I)| \\
& \leq\left|\sum_{\xi \in I} f(\xi)\right| I|-F(I)|+\left|\sum_{2} f\left(\xi^{\prime}\right)\right| I|-F(I)|+\left|\sum_{1} f(\xi)\right| I|-F(I)| \\
& +\left|\sum_{2}\left(f(\xi)-f\left(\xi^{\prime}\right)\right)\right| I|| .
\end{aligned}
$$

The total of the first two summations is less than $\epsilon$, since $(I, \xi),\left(I, \xi^{\prime}\right)$ form a partial $\delta$-fine division, with $\xi \in I, \xi^{\prime} \in I$. The subsummation $\sum_{1}$ is less than $2 \epsilon$, by Corollary 1. For the last summation, $\left|\sum_{2}\left(f(\xi)-f\left(\xi^{\prime}\right)\right)\right| I|| \leq \sum_{2} \epsilon|I| \leq$ $\epsilon|E|$ since $\xi, \xi^{\prime} \in X(\epsilon, k, n, q)$ so that $\left|f(\xi)-f\left(\xi^{\prime}\right)\right|<\epsilon$. Hence $f$ is McShane integrable on $E$. 


\section{References}

[1] Chew Tuan-Seng, Another proof that every absolutely Henstock integrable function is McShane integrable, Functional Analysis and Global Analysis, Proceedings of the Conference, Manila, October, (1996) 58-61.

[2] Wang Pujie and Lee Peng Lee, Every absolutely Henstock integrable function is McShane integrable, Journal of Mathematical Study, 27, No 2, (1994) 47-51. 